\title{
An introduction to the UCSD PASCAL system
}

\author{
KENNETH L. BOWLES \\ Institute for Information Systems, University of California, San Diego, La Jolla, California 92093 \\ and \\ JAMES D. HOLLAN
Center for Human Information Processing, University of California, San Diego \\ La Jolla, California 92093 \\ and Navy Personnel Research and Development Center, San Diego, California 92133
}

\begin{abstract}
This paper provides an introduction to the UCSD PASCAL system: a nearly machineindependent software system for micro- and minicomputers. The system is designed to run as a single-user interactive system. It is written almost entirely in the PASCAL programming language and consists of: a single-user operating system, PASCAL pseudomachine interpreter, PASCAL compiler, screen- and line-oriented editors, file manager, debugger, utility programs, and a BASIC compiler. A CAI package is also available.
\end{abstract}

Computers are getting smaller, and cheaper, and faster, and more reliable, and less energy demanding. Everything is changing together in the right direction. The good things do not trade off against the bad ones. More speed does not mean more dollars. Smaller size does not mean lower reliability. On any given date, the expected painful tradeoffs do hold, just as we learned in elementary economics. It costs more to buy faster circuits or larger memories. But come back next year and everything is better, smaller, cheaper, faster, more reliable, less energy. - Allen Newell

The monotonically decreasing costs of mini- and microprocessors has been accompanied, almost paradoxically, by increasing processor power and speed of execution. This decreasing cost and increased power has resulted in these systems being considered as replacements for earlier more expensive processors, as alternative sources for services previously obtained from large timesharing systems, and for use in a growing number of special-purpose applications. The major complications to this otherwise very pleasant state of affairs have been the ever-increasing costs of software development and the frequent unavailability of highlevel languages for the new processors. Thus, as in the past, although hardware costs are declining, it remains difficult to move existing software to new machines and expensive to develop software for new applications.

The use of high-level languages and structured programming techniques has gained acceptance for most programming applications from the development of specific user application programs to the implementation of operating systems and other systems-level programs.

This research was supported by Contract N00123-76-C-1546 from the Navy Personnel Research and Development Center. The views expressed are those of the authors and should not be construed as official or reflecting the views of the Navy Department. Requests for reprints should be sent to James D. Hollan, Department of Psychology C-009, University of California at San Diego, La Jolla, California 92093.
A challenge that presents itself is to allow program development to be accomplished in a high-level language, with all of its associated virtues, but still maintain machine independence for the resulting software.

In the past, the typical approach has been to make use of widely available languages such as FORTRAN and assume that such languages, because of their widespread use, would be made available on new processors. This has in general been the case with largescale computers, but even here there are many problems associated with transporting a program from one operating system to another. With smaller machines, there has not been an availability of common high-level languages.

The approach discussed in this paper involves the design of a development system that allows the use of a high-level language for system and program development but reduces machine dependence by producing pseudomachine code that will run on any processor provided with an interpreter for the pseudomachine code. A pseudomachine interpreter is a program, usually written in the machine language of a host machine, that emulates the operation of the pseudomachine. Typically, the interpreter executes pseudomachine code produced by a high-level language compiler. A major advantage of this approach is that the pseudomachine code can be executed on any machine provided with a pseudomachine interpreter. Note, though, that, as is the case with the system described in this paper, if an operating system itself is written in the high-level language and runs on the interpreter, it can also be moved just as easily from machine to machine. Thus, this approach not only allows the transfer of individual application programs from one machine to another, it also allows the transfer of the complete development system itself in the same manner. 


\section{THE UCSD PASCAL PROJECT}

The UCSD PASCAL project, under the direction of Ken Bowles, is an activity of the Institute for Information Systems. Among the main objectives of the project are the following: (1) To foster widespread use of machine-independent software systems, particularly for small computers, as a means of avoiding software obsolescence; (2) to promote the widespread use of PASCAL as a general-purpose programming language, both for writing system programs, such as operating systems and compilers, and for applications software in education, research, and business; (3) to foster the development of a national or international market place within which authors of computer-based course materials and other applications software may receive reasonable royalties to compensate them for their work; (4) to demonstrate that it is practical to improve the quality of mass education at the college level, while simultaneously reducing cost, through the use of microcomputer-based course materials coupled with Keller's Personalized System of Instruction.

\section{THE PASCAL SYSTEM}

The UCSD PASCAL system is a complete interactive software system for small computers, yet it offers many features normally found only on mediumand large-scale machines. It is designed to operate, with minimal adaptation, on most micro- and minicomputers based on 8-bit bytes and/or 16-bit words. Supported versions are now available for use on machines based on the Digital Equipment Corporation (DEC) LSI-11 or other PDP-11 processors, and on the 8080 and $\mathrm{Z} 80$ microprocessors. Having first been sent to users in August 1977, the system is now in use on approximately 60 mainframes using these processors, and the list of users is growing rapidly. It is expected that versions of the system will shortly be supported for operation on four additional processors (General Automation 440, Univac V75, Nanodata QM-1, and National Semiconductor PACE).

The system has been written almost entirely in the PASCAL programming language (Jensen \& Wirth, 1974), which has been extended (Bowles, 1977) for system programming and disk-based interactive applications. It is a complete and fully integrated system that is self-maintaining and generally independent of software from any other source. The system operates on a small pseudomachine interpreter that can be written in the native language of conventional processors or can be microprogrammed on machines that provide that capability. The object code processed by the PASCAL pseudomachine is compressed relative to conventional object code and consumes approximately one-third to one-half as much space as the native object code of most present-day processors. A feature planned for future implementation will allow mixing PASCAL pseudocode routines, for efficient use of memory, with native code routines, for fast processing.

The major components of the system currently being distributed include the following: single-user operating system, PASCAL pseudomachine interpreter, PASCAL compiler, screen- and line-oriented editors, file manager, debugger, utility programs, a BASIC compiler, and a CAI package.

\section{The Development System}

The UCSD PASCAL system has been designed to run as a single-user interactive system with superior response characteristics when used with floppy-disk secondary storage. Much of the development work was accomplished on Terak microprocessor systems. The Terak system includes a keyboard, bit-mapped graphics (240 x 320) CRT, LSI-11 CPU, $28 \mathrm{~K}$ words of memory, serial interface, and a single floppy-disk drive. This system is available through EDUCOM for about $\$ 5,500$.

\section{Characteristics of Host Machines}

While the system has proven that machine independence of a complex software system is practical, There are, of course, limits to the range of characteristics that can be accommodated in the host machine. This section summarizes the important characteristics of suitable host machines.

If one wishes to run the compiler for the full standard PASCAL language, plus UCSD extensions, minimum memory size is $48 \mathrm{~K}$ contiguous 8 -bit bytes, even for compiling small programs. Most of the development work was accomplished on LSI-11 machines with $56 \mathrm{~K}$ bytes of memory, and this has proven more than adequate except when compiling very large programs. The interpreter uses about $8 \mathrm{~K}$ bytes, which on some machines could be in ROM or microcode. The resident operating system requires about $8 \mathrm{~K}$ bytes. It is expected that shortly it will be possible to configure specialized systems that require substantially less than $16 \mathrm{~K}$ bytes of resident space. Users who do not wish to use the system for compiling will find it practical to operate with $16 \mathrm{~K}$ bytes (and eventually less) of resident memory, plus sufficient memory for their particular application program. The system is not designed to run on a machine with its main memory broken into two or more noncontiguous areas, since input-output (I/O) handlers and other special functions are allocated to the middle of the usual $65 \mathrm{~K}$-byte address range.

The system is designed to operate on machines that use 16-bit memory words and 8-bit bytes. The 16-bit word size is simulated on the 8080 and similar microprocessors. The attempt to extend the concept of machine independence to machines with other effective word sizes is deemed impractical and a large effort would be required to alter the system to work on such machines.

Currently, the only secondary storage medium fully supported by the project is the IBM 3740-compatible 
floppy disk. A few copies of the system have been distributed on DEC RKO5-compatible hard disks. Blocking in both cases is compatible with the 512-byte blocks used in DEC's RT-11 operating system, and this extends to alternate mapping of 128-byte sectors on the floppy disks. Other media and formats will eventually be supported. It is interesting to note that the transfer effort involved in enabling the system to operate in other secondary storage environments has often proven to be more than that required to convert from one processor to another.

It is very probable that arrangements will be made to ship copies of the system for use on floppy disks in the new quad-density format (1.2 million bytes/disk, 1,024 byte sectors), recently announced by IBM, and in the standard density format on hard-sectored disks. It is also possible to ship copies of the system on sevenand nine-track standard computer tape written at 800 bpi.

The system makes extensive use of the disk medium for reading in overlaid code files, file directories, error messages, and other frequently needed information. Because of this, it would be impractical to use magnetictape cassettes or cartridges for storing the system software.

The code files are reentrant and, in principle, could be stored in paged read-only memory. This would yield substantially faster shifting between major system modules than is possible with floppy disks, but it would impose difficult user-support problems in view of the rate at which the system is currently evolving. At present, the disk space occupied by the major modules of the system is already $65 \mathrm{~K}$ bytes.

The system has been designed with the philosophy that the principal user interface will be via a keyboard with fast $(9,600$ baud) ASCII CRT terminal. In most cases, single-character commands are used in conjunction with prompting messages to remind the user of the various commands that are available in a given context. It is assumed that, in addition to the normal return and line-feed functions, the CRT display has hardware functions for moving the cursor to any row/column position (cursor addressability), and for erasing from the current cursor position to the end of line. It is assumed that the display is at least 80 columns wide and 24 lines high. Other console output devices can be accommodated, although the results are not always aesthetically pleasing. A simple editor, similar to that provided with DEC's RT-11 operating system, is provided for use with line-oriented terminals such as teleprinters.

The system makes extensive use of control codes normally represented by the ASCII characters CR, ESC, ETX, BS, DEL, and TAB, as well as the BREAK key. If the main screen-oriented editor will be used, position arrow keys (up, down, right, left) are also needed, as is a mechanism for key-stroke repetition. Means are provided to set up the system for regular use of alternatives for all these keys. It is assumed that all 95 of the visible characters can be generated from the keyboard, with the possible exception of lowercase letters.

The system provides a standard mechanism for writing out data to a printer file, for output and input for a remote communications file, and potentially to other standard interface files. The printer driver provided with the PDP-11 version of the system is designed to drive a Printronix 300 printer with Dataproducts oriented interface. This driver can serve as a model for the development for other devices.

\section{APPLICATIONS AND AVAILABILITY}

The LCSD PASCAL system began as a simple system intended primarily for development of small student programs and for interactive instructional applications (CAI). It has evolved into a general-purpose software development system for micro- and minicomputers.

At UCSD, the software system is being used with Keller's Personalized System of Instruction (PSI) in large-enrollment (500 students per quarter) introductory computer science courses. The unusual effectiveness attributed to PSI relies heavily on individual attention to students by teaching aids known as "proctors." This reliance makes the typical PSI course, without computers, somewhat more labor intensive than conventional college courses. We have found that the use of microcomputers to handle many routine aspects of the duties normally assigned to the proctors (quizzes, drill and practice, and recordkeeping) allows both an increase in the attention given to individual students and a substantial reduction in labor costs. Instructional applications of this type should be readily applicable to mass education of high quality and low cost in many fields at the college level.

Currently, efforts are underway to use the UCSD PASCAL system and CAI package to automate quiz administration for a large introductory psychology course. Software is also being developed to allow students in a laboratory course in cognitive psychology to perform a wide range of experiments concerned with the investigation of the dynamics of mental processes. In addition, a set of PASCAL programs is being implemented to assist students in statistical and other quantitative methods courses offered by the psychology department. The programs will allow students to collect data from simulated experiments and permit statistical analysis of the data. The goal is to create an environment in which a student can gain experience in the analysis of data while simultaneously being freed from much of 
the tedium involved in collecting data and performing statistical analyses.

The UCSD PASCAL system is available for a distribution fee of $\$ 200$. Interested individuals should contact the UCSD PASCAL Project, Institute for Information Systems, University of California at San Diego, La Jolla, California 92093.

\section{REFERENCES}

BOWLES, K. L. Microcomputer problem solving using PASCAL. New York: Springer-Verlag, 1977.

JENSEN, K., \& WIRTH, N. PASCAL user manual and report. New York: Springer-Verlag, 1974.

(Received for publication March 21, 1978; revision accepted June 19,1978 .) 